\title{
Complex Geometry of Wave Motion
}

\author{
Eugene Machusky
}

\begin{abstract}
All fundamental constants of quantum physics are identified as geometrical parameters of a spherical spiral rhythmically pulsating from zero to infinity relatively of equator $P I * E$. This product of transcendental numbers generates functions of normal distribution of inverse radii and corresponding eccentricities and perimeters. Gradients of both normal and log-normal distribution of reversed geometrical parameters give possibility to identify and evaluate physical quantum units with practically unlimited accuracy. Application of parabolic, hyperbolic and logarithmical bonds of transcendental numbers PI and $E$ solves the problem of geometrical commutativity and mutually coordinates the quantum constants. For the first time the expressions for units of Kelvin, Avogadro, Boltzmann, Planck, of speed of light, background temperature, harmonic translational velocity, of fine structure, elementary charge, relative molar mass and Newtonian gravitation have been obtained analytically. Quantum units are mutually matched on accuracy within most accurate CODATA values. The main conclusion is that quantum physics, in fact, is a two-dimensional image of a wave front motion in a three-dimensional space.
\end{abstract}

Index Terms -Absolute metric, noncommutative mathematics, complex geometry, gauge equations, fundamental quantum constants.

\section{INTRODUCTION}

The first attempt to develop an absolute metric system of physical units was made by Gauss at the beginning of nineteenth century. The attempt was unsuccessful because of an inconsistency of hyperbolic and elliptic geometry [1]. Application of parabolic, hyperbolic and logarithmic bonds of transcendental numbers PI and $\mathrm{E}$ gives a possibility to solve the problem of geometrical inconsistency and to coordinate the quantum constants [2]. The main shortcoming of contemporary metric systems is an insufficiently exact value of relative molar mass unit because the least mistake at the third place of decimal fraction can produce one-percent deviation of calculation result. Announced by NIST the New SI measurement system [3] inherited the same disadvantage. The problem can be solved only and only by the means of analytical derivation and mutual coordination of units of temperature, speed, charge and mass [4].

\section{INVERSE GEOMETRY}

Mutual bonds of geometrical parameters of pulsing sphere can be described by a single expression:

$$
[\mathrm{Gi}]=2 * \mathrm{PI} *[\mathrm{Ri}]^{*}\{1+[\mathrm{Ai}]\},
$$

Manuscript received May 9, 2017; revised July 13, 2017.

Eugene Machusky is with National Technical University of Ukraine "Kyiv Polytechnic Institute", Ukraine (e-mail: sivera@ukr.net). where [Ri] is a matrix of relative inverse radii, [Ai] is a matrix of relative inverse eccentricities, $2 * \mathrm{PI} *[\mathrm{Ri}]=[\mathrm{Pi}]$ is a matrix of relative inverse perimeters. The instant values of radii $\mathrm{Ri}$ and eccentricities $\mathrm{Ai}$ are linked by the parameter $\operatorname{sqrt}(2 * \mathrm{PI} * \mathrm{E})$ of the Gaussian function of normal distribution:

$$
\begin{gathered}
\mathrm{Ri}=1+2 / 100 *(\mathrm{E}+\mathrm{Ai} *(1+\operatorname{sqrt}(2 * \mathrm{PI} * \mathrm{E}) / 10)), \\
\mathrm{Ai}=(100 *(\mathrm{Ri}-1) / 2-\mathrm{E}) /\left(1+\operatorname{sqrt}\left(2 * \mathrm{Pi}^{*} \mathrm{E}\right) / 10\right) .
\end{gathered}
$$

The inverse eccentricities are concentrated near reciprocal value of squared sum of mean values (root mean square, arithmetical mean, geometrical mean and harmonic mean) of numbers PI and E:

$$
1 /\left\{\operatorname{sqrt}\left[\left(\mathrm{PI}^{\wedge} 2+\mathrm{E}^{\wedge} 2\right) / 2\right]+(\mathrm{PI}+\mathrm{E}) / 2+\operatorname{sqrt}(\mathrm{PI} * \mathrm{E})\right.
$$

$+2 * \mathrm{PI} * \mathrm{E} /(\mathrm{PI}+\mathrm{E})\}^{\wedge} 2=1 / 136.9938985020083597$.

An exponential bond of PI and E is illustrated by the expression:

$$
\mathrm{PI}^{*} 10^{\wedge} 59=\mathrm{E}^{\wedge} 136.9972503724980956 .
$$

Averages of reciprocal values of these digital sequences begin to differ of the 12th decimal place. This is the natural limit of accuracy of geometrical calculations in inverse polar coordinates, if PI and E are base of calculation system. It might be that this is the key to the noncommutative mathematics [5].

\section{CAlibrating EQUATIONS}

\section{A. Electrodynamics}

Two equations constitute basis of the Maxwell-Gauss electrodynamics:

$\mathrm{C}=\left(\mathrm{R}+4 * \mathrm{PI} * \mathrm{C} / 10^{\wedge} 18\right)^{\wedge} 64 * 10^{\wedge} 7=$ 299792457.867591338433684,

$$
\mathrm{R}=\text { Integer }\left[10^{\wedge} 8^{*}\left(\mathrm{C} / 10^{\wedge} 7\right)^{\wedge}(1 / 64)\right] / 10^{\wedge} 8=1.05456978 .
$$

The number $\mathrm{C}$ is a value of Rotational speed of radius-vector in the $3 \mathrm{D}$ space. The number $\mathrm{R}$ is a length of the corresponding inverse squared radius. The value $4 * \mathrm{PI}$ is the Solid angle in radians. The value $4 * \mathrm{PI}^{*} 10^{\wedge}-7$ is the Magnetic constant. The value $4 * \mathrm{PI} * \mathrm{C}^{*} 10^{\wedge}-7$ is the Impedance of free space. The value $1 /\left(4^{*} \mathrm{PI}^{*} 10^{\wedge}-7\right) / \mathrm{C}^{\wedge} 2$ is a lower limit of the Electric constant.

Number $\mathrm{C}$ is the Speed of light in physics, but arithmetically it is simply a quantity of turns around a ball with the radius $4 * \mathrm{PI} / 10^{\wedge} 18$ during a unit of time.

\section{B. Thermodynamics}

Three equations constitute basis of the Kelvin-Avogadro thermodynamics: 


$$
\mathrm{K}=\mathrm{E}+\mathrm{AS}+\mathrm{BS}=2.7315999984590452 .
$$

AS $=1 / 100 /$ Sum $\left\{[137+\{137100) * n] / 10^{\wedge}(3 * n+2)=\right.$ $0.00729=1 / 100 /(1.11111111111 \ldots)^{\wedge} 3$.

BS $=$ Sum $\left[602214183 / 10^{\wedge}(3 * \mathrm{n}+8)\right]=$ $0.00602816999999 \ldots 999999397183=0.00602817$.

The number K (kelvin) is an upper limit of an inverse temperature that is used in SI for calibration of triple point of water as $100 * \mathrm{~K}=273.16$. The word "Temperature" literally means "rating of tempo" or, in other words, "rating of velocity of vibrations". Instant value of translational velocity oscillates between the values of rotational and vibrational speeds.

Numbers AS and BS link "two letters alphabet" $(0 \ldots 1)$ and "ten letters alphabet" $(0 \ldots .9)$ calculation systems because $1=$ $(0.999999 \ldots)$ and $10=(9.99999 \ldots)$ [6].

The number $\mathrm{B}=602214183$ is the Avogadro's integer.

The sequence PI = Sum $\{$ from $(-1)$ to $(+1)\}$ of $\left[\mathrm{dx} / \mathrm{sqrt}\left(1-\mathrm{x}^{\wedge} 2\right)\right]$, where $\mathrm{x}$ tends to Zero, is equal to 3.1415926535897932 , which is a perimeter of the circle with the diameter equal to 1 .

The sequence $\mathrm{E}=(1+1 / \mathrm{n})^{\wedge} \mathrm{n}$, where $\mathrm{n}$ tends to Infinity $=$ 2.7182818284590452 , is the base of a natural logarithm in mathematics, but in physics it means a lower limit of an inverse temperature.

\section{QUANTUM CALIBRATING FIELDS}

Basic quantum calibrating geometrical ("Gauge") field (Fig.1) has been constructed as the matrix of inverse squared radii:

$\mathrm{R}+4 * \mathrm{PI} * \mathrm{C} / 10^{\wedge} 18=1.0545697837673031=\mathrm{R} 8=\mathrm{RC}$.

$\mathrm{R}+1 / \mathrm{E} / 10^{\wedge} 8=1.0545697836787944=\mathrm{R} 7=\mathrm{RE}$.

$\mathrm{R}+1 /(\mathrm{E}+\mathrm{AS}) / 10^{\wedge} 8=1.0545697836608580=\mathrm{R} 6=\mathrm{RA}$.

$\mathrm{R}+1 /(\mathrm{E}+\mathrm{AS}+\mathrm{BS}) / 10^{\wedge} 8=1.0545697836608580=\mathrm{R} 5=\mathrm{RK}$.

$\mathrm{R}-1 /(\mathrm{E}+\mathrm{AS}+\mathrm{BS}) / 10^{\wedge} 8=1.0545697763391420=\mathrm{R} 4$.

$\mathrm{R}-1 /(\mathrm{E}+\mathrm{AS}) / 10^{\wedge} 8=1.0545697763310450=\mathrm{R} 3$.

$\mathrm{R}-1 / \mathrm{E} / 10^{\wedge} 8=1.0545697763212056=\mathrm{R} 2$.

$\mathrm{R}-4 * \mathrm{PI}{ }^{*} \mathrm{C} / 10^{\wedge} 18=1.0545697762326969=\mathrm{R} 1$.

This Gauge field can be identified as the Heisenberg uncertainty matrix.

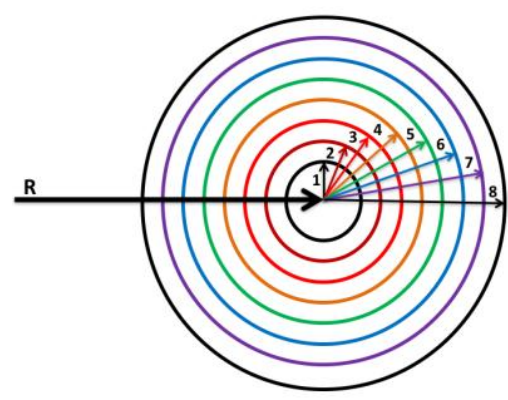

Fig. 1. Geometry of rotating radius-vectors

TABLE I: LOWER PART OF RADIAL FIELD

\begin{tabular}{lll}
\hline Radius & Value & Description \\
\hline RS & 1.0545716917923240 & R(AS) \\
RVS & 1.0545707377551176 & Median (RV; RS) \\
RM & 1.0545688734791973 & Median (RVX; RVS) \\
RC & 1.0545697837673031 & R+4*PI*C/10^18 \\
RV & 1.0545697837183468 & Median (RT; RC) \\
RE & 1.0545697836787944 & R+1/E/10^8 \\
RAE & 1.0545697836738746 & Median (RA; RE) \\
RT & 1.0545697836693905 & Median (RAK; RAE) \\
RA & 1.0545697836689549 & Median (RA; RK) \\
RAK & 1.0545697836649065 & Median (RA; RK) \\
RK & 1.0545697836608581 & R+1/K/10^8 \\
RVX & 1.0545670092049249 & Median (RX; RV) \\
RX & 1.0545642346951568 & R(AX) \\
\hline
\end{tabular}

The referenced above value $\mathrm{AX}=5 / \mathrm{X}-1=$ 0.0070261763632109 , where $\mathrm{X}=$ Root of $\left[\mathrm{X}^{*} \mathrm{E}^{\wedge} \mathrm{X} /\left(\mathrm{E}^{\wedge} \mathrm{X}-1\right)\right.$ $=5]=4.9651142317442763$. This value is known as a parameter of equation for Wien wavelength displacement of blackbody irradiation [7].

TABLE II: UPPER PART OF RADIAL FIELD

\begin{tabular}{lll}
\hline Radius & Value & Description \\
\hline R1 & 1.0545719538152265 & R(A1) \\
RF & 1.0545718996147182 & R(AF) \\
R01 & 1.0545718610477856 & Median (R0; R1) \\
RQ & 1.0545718475783874 & Median (RP; RF) \\
RP & 1.0545717955420578 & Median (R0S; R01) \\
R0 & 1.0545717682803448 & R(A0) \\
R0S & 1.0545717300363340 & Median (R0; RS) \\
RS & 1.0545716917923240 & R(AS) \\
\hline
\end{tabular}

The referenced above sequence $\mathrm{A} 1=1 / 137=$ $0.0072992700729927 \ldots$ is the parameter of a mirror symmetry of an inverse natural set. The referenced above sequence $\mathrm{AF}=1 / \operatorname{Integer}\left[1000^{*} \operatorname{sqrt}\left(137^{\wedge} 2+\mathrm{PI}^{\wedge} 2\right)\right] / 1000=$
0.0072973525205056 links numbers 137 and PI, the sequence $\mathrm{A} 0=(\mathrm{PI} * \mathrm{E} / 100)^{\wedge} 2=0.0072927060593902$ binds the numbers PI and $\mathrm{E}$. 
TABLE III: INFORMATION ENTROPY FIELD

\begin{tabular}{lll}
\hline Entropy & Value & Description \\
\hline N4 & 0.0060221410025819227 & $\mathrm{~N}(\mathrm{~A} 4)$ \\
NH4 & 0.0060221410039861000 & Median $(\mathrm{NH} ; \mathrm{N} 4)$ \\
NH & 0.0060221410053902884 & $\mathrm{~N}(\mathrm{AH})$ \\
$\mathrm{NB}$ & 0.0060221410732354338 & $\mathrm{~B} /\left(1+4 * \mathrm{PI} / 10^{\wedge} 8\right) / 10^{\wedge} 11$ \\
NN & 0.0060221410745006000 & $\mathrm{Median}(\mathrm{NL} ; \mathrm{NH} 4)$ \\
NL & 0.0060221411450151730 & $\mathrm{~N}(\mathrm{AL})$ \\
\hline
\end{tabular}

The referenced above sequence $\mathrm{A} 4=(\mathrm{PI} * \mathrm{E} / 100)$ ${ }^{\wedge} 2+4 *\left[1 / 137-(\mathrm{PI} * \mathrm{E} / 100)^{\wedge} 2\right]=0.0073189621138002$ and the sequence $\mathrm{AH}=1 /\left(4^{\wedge} 2^{*} \mathrm{PI} * \mathrm{E}\right)=0.0073187289405399$ arithmetically link the numbers $\mathrm{PI}$ and $\mathrm{E}$. The sequence $\mathrm{AL}=$
$1 /[1+59 * \ln (10)]$ logarithmically binds 10 and $\mathrm{E}$. The set of sequences $\mathrm{Ni}=\left\{\operatorname{sqrt}\left[8^{*} \mathrm{PI} * \mathrm{E} /\left(8 * \mathrm{PI} * \mathrm{E}+137^{\wedge} 2\right)\right] /\right.$ $(1+2 *[$ AL ...A4 $\left.] / 1000)-5 / 10^{\wedge} 8\right\} / 10$ coordinates arithmetical and logarithmical bonds of transcendental numbers PI and E.

TABLE IV: HARMONIC TRANSLATION VELOCITY

\begin{tabular}{lll}
\hline Velocity & Value & Description \\
\hline $\mathrm{C}$ & $2.9979245787659074 * 10^{\wedge} 8$ & $\mathrm{RC}^{\wedge} 64 / 10$ \\
& $2.9979245697688564 * 10^{\wedge} 8$ & Root mean square \\
$\mathrm{V}$ & $2.9979245697688564 * 10^{\wedge} 8$ & Arithmetical mean \\
& $2.9979245697688564 * 10^{\wedge} 8$ & Median $(\mathrm{T} ; \mathrm{C})$ \\
$\mathrm{T}$ & $2.9979245697688564 * 10^{\wedge} 8$ & Geometrical mean \\
& $2.9979245697688564 * 10^{\wedge} 8$ & Harmonic mean \\
$\mathrm{RT}^{\wedge} 64 / 10$
\end{tabular}

\section{BASIC QUANTUM UNITS}

The value of translational velocity $\mathrm{V}$ oscillates between the values of rotational speed $\mathrm{C}$ and vibrational speed $\mathrm{T}$. All mean values of $\mathrm{V}$ are mutually equal, which is impossible in continuous mathematics, but this is an indefeasible result produced in the discrete mathematics with the limited capacity of display.

\section{A. Harmonic Planck Unit}

The value of Planck constant is identified as the harmonic perimeter $\mathrm{H}$ :

$2 * \mathrm{PI} * \mathrm{R} 01=6.6260704227004664=\mathrm{P} 01$

Median $(\mathrm{P} 0 \mathrm{~S} ; \mathrm{P} 01)=6.6260700111158525=\mathrm{H}$.

Actual CODATA value is 6.626070040(81).

$2 * \mathrm{PI} * \mathrm{R} 0 \mathrm{~S}=6.6260695995312512=\mathrm{P} 0 \mathrm{~S}$

\section{B. Fine Structure Constant}

The value of Fine structure constant is identified as $\mathrm{AF}=$ $1 /$ Integer[1000*sqrt $\left.\left(137^{\wedge} 2+\mathrm{PI}^{\wedge} 2\right)\right] / 1000$ 0.0072973525205056 . CODATA conventional value $0.0072973525664(17)$ is not correct at 12 th place.

\section{Harmonic Elementary Charge Unit}

The value of Elementary charge (charge of electron) is identified as the harmonic density of orbital translational velocity Q (in accordance with the Standard physical model): Sqrt $\left(10 * \mathrm{AF}^{*} \mathrm{RP} / \mathrm{T}\right)=1.6021766174049711=\mathrm{QT}$.

Median $(\mathrm{QT} ; \mathrm{QC})=1.6021766150248797=\mathrm{Q}$.

Last CODATA value is 1.6021766208(98).

Sqrt $\left(10 * \mathrm{AF}^{*} \mathrm{RP} / \mathrm{C}\right)=1.6021766126198929=\mathrm{QC}$.

\section{Harmonic Molar Mass Unit}

The value of a molar mass MM is identified as the time-corrected number of "kisses" of each element of ideal gas during one turn between the 12 "neighbors":

$12-\mathrm{AT} / 10=11.9992777507224389=\mathrm{MMT}$.

Median $($ MMC; MMT) $=11.9992777505492373=$ MM.

Now CODATA value is 12 (carbon-12).

$12-\mathrm{AC} / 10=11.9992777503760353=$ MMC.

\section{E. Harmonic Boltzmann Unit}

The value of Boltzmann constant $\mathrm{KB}$ is identified as the harmonic phase of rotating radius-vector:

$\cos ($ MMT $)-\sin ($ MMT $)=1.380648450284=\mathrm{KBT}$.

Median $(\mathrm{KBC} ; \mathrm{KBT})=1.380648450231=\mathrm{KB}$.

CODATA value is $1.38064852(79)$.

$\cos (\mathrm{MMC})-\sin (\mathrm{MMC})=1.380648450177=\mathrm{KBC}$.

\section{F. Harmonic Avogadro Unit}

The value of Avogadro constant NA is identified as the harmonic value of entropy of calculations of geometrical parameters:

$\mathrm{NN}^{*} 1000=6.0221410745006000$.

Median $(\mathrm{NB} ; \mathrm{NN})=6.0221410738680000=\mathrm{NA}$.

Last CODATA value is 6.022140857(74).

$\mathrm{NB} * 1000=6.0221410732354338$.

\section{G. Harmonic Newtonian Gravitational Unit}

The value of Newtonian gravitational constant $G$ is identified as the value of harmonic density of the velocity field:

$2 * \mathrm{PI} * \mathrm{RQ} *(1+\mathrm{AQ})=6.6744109106473266=\mathrm{GQ}$.

Median $(\mathrm{GM} ; \mathrm{GQ})=6.6740529010349328=\mathrm{G}$.

CODATA value is 6.67408(31).

$2 * \mathrm{PI} * \mathrm{RM} *(1+\mathrm{AM})=6.6736948926580828=\mathrm{GM}$.

\section{COMPLEX VERIFICATION}

The following below shows that an excellent complex verification of a mutual coordination of quantum units:

Molar gas constant (thermodynamics): $\mathrm{KB} * \mathrm{NA}=$ 8.3144597407083043 .

CODATA value is $8.3144598(48)$.

Wien wavelength displacement (quantum physics): $2 * \mathrm{PI} * \mathrm{RP} * \mathrm{~V} / \mathrm{KB} / \mathrm{X} \quad=\quad 0.028977730384396$ CODATA value is $0.028977729(17)$. 


\section{DECIMAL ORDERS OF QUANTUM CONSTANTS}

Decimal orders of quantum constants are determined from the equations of the standard physical model using an approximate exponential expression $\mathrm{E}^{\wedge} 137=100 * \mathrm{PI}^{*} 10^{\wedge} 57$. For KB this value is $10^{\wedge}-23$. For NA it is $10^{\wedge}+23$.

For RP and $H$ the value of a decimal order is $10^{\wedge}-(57-23)$ $=10^{\wedge}-34$. For $\mathrm{Q}$ it is $10^{\wedge}-(8+11)=10^{\wedge}-19$ and for $\mathrm{G}$ it results in $10^{\wedge}+(8-19)=10^{\wedge}-11$.

\section{GAUGE EQUATIONS AND DIGITAL SEQUENCES}

The values for the constants for the standard measurements could be identified as presented below.

The Absolute Space Number (perimeter of circle with diameter 1):

$\mathrm{PI}=$ Sum from $(-1)$ to $(+1)$ of $\left\{\mathrm{d}(1 / \mathrm{N}) / \operatorname{sqrt}\left[1-(1 / \mathrm{N})^{\wedge} 2\right]\right\}$, where $\mathrm{N}$ tends to Infinity, identifies the value of

$\mathrm{PI}=31415926535897932 / 10^{\wedge} 16$

The Absolute Time Number (a base of natural logarithm, lower limit of an inverse temperature):

$\mathrm{E}=(1+1 / \mathrm{N})^{\wedge} \mathrm{N}$, where $\mathrm{N}$ tends to Infinity, identifies the value of

$\mathrm{E}=27182818284590452 / 10^{\wedge} 16$

Maxwell number (an upper limit of harmonic rotational speed)

$\mathrm{C}=\left(\mathrm{R}+4 * \mathrm{PI}{ }^{*} \mathrm{C} / 10^{\wedge} 18\right)^{\wedge} 64^{*} 10^{\wedge} 7$ identifies the value of $\mathrm{C}=\mathrm{C}=299792457.8675913384336840 / 10^{\wedge} 16$

Dirac number (a harmonic inverse nuclear radius)

$\mathrm{R}=$ Integer of $\left[\left(\mathrm{C} / 10^{\wedge} 7\right)^{\wedge}(1 / 64)^{*} 10^{\wedge} 8\right] / 10^{\wedge} 8$ identifies the value of $\mathrm{R}=1.05456978$

Kelvin number (an upper limit of inverse temperature)

$\mathrm{K}=\mathrm{E}+\mathrm{AS}+\mathrm{BS}$ identifies the value of $\mathrm{K}=27315999984590452 / 10^{\wedge} 16$

Where the definitions of the following values are taken as below:
AS - the Absolute Symmetry Unit

AS $=1 /$ Sum of $\left\{\left[137+(137-100)^{*} \mathrm{~N}\right] /\left[10^{\wedge}\left(3^{*} \mathrm{~N}\right)\right]\right.$, where an integer $\mathrm{N}$ tends from Zero to Infinity, is identified by the value $729 / 10^{\wedge} 5$ deducted in the different ways as below:

AS $=1 / 100 /(10 / 9)^{\wedge} 3=3 * 3 * 3 * 3 * 3 * 3 / 2 * 2 * 2 * 2 * 2 / 5 * 5 *$

$* 5 * 5 * 5=3^{\wedge} 6 / 10^{\wedge} 5=0.00729=729 / 10^{\wedge} 5$

$\mathrm{AS}=1 /(100) /(1.111111111111111111111111111$

$111111111111111111111111 \ldots)^{\wedge} 3=729 / 10^{\wedge} 5$;

AS $=\left(10^{\wedge} 0\right) /\left(10^{\wedge} 2\right) /$

$\left[\left(10^{\wedge} 0\right)+\left(10^{\wedge}-1\right)+\left(10^{\wedge}-2\right)+\left(10^{\wedge}-3\right)+\left(10^{\wedge}-4\right)+\left(10^{\wedge}-5\right)+\left(10^{\wedge}-6\right)\right.$

$+\ldots]^{\wedge} 3=729 / 10^{\wedge} 5$;

Note. [11111111111111111111111111 ..> is Schrodinger Qubit number.

$\mathrm{A}=137$ is Sommerfeld-Born-Eddington-Feynman Magic Prime

While $1 / 137=0.007299270072992700729927 \ldots$ - is a unique center of mirror symmetry of inverse natural set.

Information Entropy Reference Unit (0.00602817)

$\mathrm{BS}=$ Sum of $\left\{\left[602214183 /\left[10^{\wedge}\left(11+3^{*} \mathrm{~N}\right]\right\}\right.\right.$, where an integer $\mathrm{N}$ tends from Zero to Infinity, identifies the value of

$\mathrm{BS}=0.0060281699999999999999999999999999$ $9999999999999999 \ldots 397183=602817 / 10^{\wedge} 8$.

The unique Avogadro integer

$\mathrm{B}=602214183$.

\section{COMPARISON OF VALUES}

New SI measurement system will be introduced at the end of year 2018 and will be based only on constants derived from nature. Up to now the main problem is to find the coordinated values of basic quantum units. This problem can be solved not only without use of artifacts (meter, kilogram, second) but at all without any measurements. Comparison of analytically obtained units with their recommended by CODATA values is presented below:

TABLE V: COMPARISON OF VALUES

\begin{tabular}{lll}
\hline Value & Experimental & Analytical \\
\hline Speed of light & 299792458 & 299792457.86759134 \\
Background temperature & 2.725 & 2.7252543275634558 \\
Relative molar mass & 0.012 & $0.0119992777505492)$ \\
Kelvin reference unit & 2.7316 & 2.7315999984590452 \\
Fine structure unit & $0.0072973525664(17)$ & 0.0072973525205056 \\
Avogadro constant & $6.022140857(74)$ & $6.0221410564201849^{*} 10^{\wedge} 23$ \\
Boltzmann constant & $1.38064857(74)$ & $1.3806484502310000^{*} 10^{\wedge}-23$ \\
Planck constant & $6.626070040(81)$ & $6.6260700111158522^{*} 10^{\wedge}-34$ \\
Elementary charge & $1.6021766208(98)$ & $1.6021766150248797 * 10^{\wedge}-19$ \\
Gravitational constant & $6.67408(31)$ & $6.6740529112548490^{*} 10^{\wedge}-11$ \\
\hline
\end{tabular}

All analytically derived values lie within measurement accuracy considering the conventionally established values of speed of light and molar mass.

\section{MiRRor Symmetry OF ClASSIC PhySICS}

All quantum units are parameters of density of the field of progressive velocity. Velocity is relative (distance unit)/ (time unit) quantity. The number $\mathrm{C}$ is upper information limit of progressive velocity and the number $\mathrm{T}$ is lower information limit of progressive velocity. It is the consequence of phase symmetry of any complex number like $\mathrm{RC}=\mathrm{R}+\mathrm{j} * 4 * \mathrm{PI} * \mathrm{C} / 10^{\wedge} 18$. All quantum constants are relative values. Kinetic energy EK $=\mathrm{m}^{*} \mathrm{v}^{\wedge} 2 / 2 *\left[1+\mathrm{v}^{\wedge} 2 /(\mathrm{T} \ldots \mathrm{C})^{\wedge} 2\right]=$ $\mathrm{m}^{*} \mathrm{C}^{\wedge} 2$ when $\mathrm{v}$ approaches $\mathrm{C}$ (Newton, Einstein), Planck energy $E P=h * f\left[1+v^{\wedge} 2 /(T \ldots C){ }^{\wedge} 2\right]=h *(2 * f)$ when $v$ approaches $\mathrm{C}$ (Kotelnikov, Nyquist), Thermal energy ET = $\mathrm{k}^{* \mathrm{~T}} /\left[1+\mathrm{v}^{\wedge} 2 /(\mathrm{T} \ldots \mathrm{C})^{\wedge} 2\right]=\mathrm{k}^{*} \mathrm{~T} / 2$ when $\mathrm{v}$ approaches $\mathrm{C}$ (Boltzmann, Shannon), Electric energy EE $=q^{*} U^{\prime}\left[1+v^{\wedge} 2 /\right.$ $\left.(\mathrm{T} . . \mathrm{C})^{\wedge} \mathrm{2}\right]=\left(\mathrm{q}^{*} / 2\right) * \mathrm{U}$ when $\mathrm{v}$ approaches $\mathrm{C}$ (Dirac, Kaufmann), where m, v, T, h, f, k, q, U are instant values of mass, velocity, action, phase, charge, tension.

\section{CONCLUSIONS}

The set of quantum units this is the 2D-image of 
3D-motion of wave fronts.

Quantum physics can be determined as the Differential geometry of pulsing spherical spiral.

Quantum physics is digital bridge between the Discrete and Continuous mathematics.

Euler's formula of continuous mathematics $E^{\wedge}(j * P I)+1=0$. Gauss' formula of discrete mathematics $\mathrm{E}^{\wedge}(\mathrm{j} * \mathrm{PI})+1=$ $1 / 10^{\wedge} 64$.

Basic formula of quantum calculus $\mathrm{E}^{\wedge}\left(\mathrm{j}^{*} \mathrm{PI}\right)+1=[\mathrm{Ai}] / 10^{\wedge} 57$.

\section{ACKNOWLEDGMENT}

E. A. Machusky thanks:

Friends: M.Sc.Ljubov Lisovska, M.Eng.Alexandr Goncharov, Dr.Victor Bocharov, for fruitful discussions and help in preparing of this article.

Colleagues: Dr. Volodimir Lutsenko, Dr.Alexandr Zhivkov, M.Eng.Volodimir Stepanenko, Dr.Sergij Zinchenko, Dr.Mykola Medvid, Prof.Alexandr Zemljac, Dr.Alexandr Progonov, Dr.Sergiy Pasichnij, Prof.Anatoly Kachinsky, Prof.Volodimir Luzhetsky, M.Eng.Olexandr Jerjomenko, for fruitful criticism and article revisions.

My wife Tatiana, M. Eng. and daughter Anna, M.Sc. - for endless patience and help.

\section{REFERENCES}

[1] Gaussian metric. en.wikipedia.org/. [Online]. Available: https://en.wikipedia.org/wiki/Gaussian_units

[2] E. A. Machusky, "Logarithmetics of quantum physics" in Proc. 16-th Int. Scientific M. Kravchuk Conf., May 14-15, 2015, Kyiv: Conf.materials. Geometry. Analysis. - K.: NTUU “KPI”, 2015. - pp. $26-29$.

[3] On the future revision of the SI. [Online]. Available: http://www.bipm.org/en/measurement-units/rev-si/

[4] roposed redefinition of SI base units. [Online]. Available: https://en.wikipedia.org/wiki/Proposed_redefinition_of_SI_base_units

[5] U. Franz and A. Skalski, Noncommutative Mathematics for Quantum Systems, Cambridge University Press, Delhi, 2016.

[6] Feynman point. [Online]. Available: http://mathworld.wolfram.com/FeynmanPoint.html

[7] Wien displacement law. [Online]. Available: https://en.wikipedia.org/wiki/Wien\%27s_displacement_law

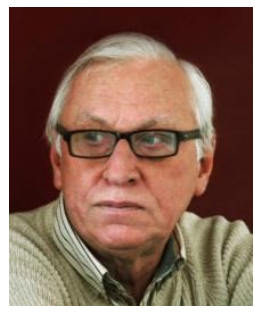

Kyiv, Ukraine.
Machusky E. was born in Lutsk, Ukraine, in 1949 He recived his M.Eng., Ph.D, D.Sc. in, 1974, 1979 1989 from NTUU"KPI", He research visitor of University of North Wales (1983-1984, Bangor, UK).

Eugene A. Machusky is a Prof. He is currently head of the Dept. of Technical Information Protection Systems, scientific Director of Special Design Bureau "Storm" in National Technical University of Ukraine "Kyiv Polytechnic Institute", 